\title{
Consumo de alcohol y tabaco en adolescentes ${ }^{1}$
}

\author{
Linda Azucena Rodríguez Puente ${ }^{2}$ \\ Bertha Alicia Alonso Castillo ${ }^{3}$ \\ Marìa Magdalena Alonso Castillo ${ }^{3}$ \\ Marìa Teresa Alonso Castillo ${ }^{3}$ \\ Nora Angèlica Armendàriz Garcìa ${ }^{3}$ \\ Nora Nelly Oliva Rodrìguez ${ }^{4}$
}

\begin{abstract}
El objetivo fue identificar el consumo de alcohol y tabaco en los adolescentes. El diseño fue descriptivo, la población estuvo conformada por 386 sujetos de entre 15 años a 20 años de una preparatoria Monterrey, Nuevo León. Los participantes respondieron una Cédula de Datos y el AUDIT. Los resultados muestran que la cantidad de bebidas consumidas en un día típico fue de 6 bebidas estándar ( $\mathrm{DE}=6.6$ ) y consumen en promedio 2 cigarrillos por ocasión de consumo ( $D E=2.4)$. El índice de bebidas alcohólicas $U=15682 ;=4.33(p=.006)$ y la cantidad de cigarrillos $U=16197 ;=1.01(p=.007)$ es mayor en hombres que en mujeres.
\end{abstract}

Descriptores: Consumo de Bebidas Alcoholicas; Uso de Tabaco; Adolescente.

\footnotetext{
${ }^{1}$ Artículo parte de Tesis de Doctorado "Valores terminales, valores instrumentales y consumo de alcohol y tabaco en estudiantes de preparatoria", presentada en la Universidad Autónoma de Nuevo León, Facultad de Enfermería, Monterrey, NL, México.

2 PhD, Profesor Asociado, Universidad Autónoma de Nuevo León, Facultad de Enfermería, Monterrey, NL, México.

${ }^{3} \mathrm{PhD}$, Profesor Titular, Universidad Autónoma de Nuevo León, Facultad de Enfermería, Monterrey, NL, México.

${ }^{4}$ Estudiante de doctorado, Profesor Asociado, Universidad Autónoma de Nuevo León, Facultad de Enfermería, Monterrey, NL, México.
}

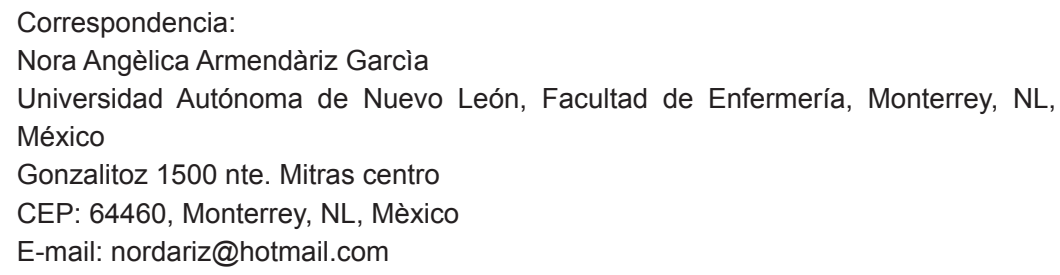




\section{Consumo álcool e tabaco entre os adolescentes}

O objetivo foi identificar o consumo de álcool e o tabaco entre os adolescentes. Trata-se de um estudo descritivo e a população foi constituída por 386 indivíduos com 15 a 20 anos de uma escola de ensino médio de Monterrey, Nuevo León. Os participantes responderam a um questionário sociodemográfico e o AUDIT. Os resultados mostram que o número de bebidas consumidas em um dia típico foi de 6 bebidas padrão ( $D E=6.6$ ) e consumiu uma média de 2 cigarros por ocasião de beber $(D E=2.4)$. Índice de bebidas alcoólicas $U=15682 ;=4.33$ $(p=0.006)$ e o número de cigarros $U=16197 ;=1.01(p=0.007)$ é maior em homens do que em mulheres.

Descritores: Consumo de Bebidas Alcoólicas; Uso de Tabaco; Adolescente.

\section{Consumption of alcohol and tobacco in adolescentes}

The aim was to identify the alcohol consumption and tobacco consumption among adolescents. The design was descriptive, the population consisted of 386 subjects aged 15 to 20 years old of Monterrey, Nuevo León high school. Participants responded to a questionary of data and AUDIT. The results show that the number of drinks consumed on a typical day was 6 standard drinks ( $S D=6.6$ ) and consumed an average of 2 cigarettes per drinking occasion $(S D=2.4)$. Alcoholic beverages index $U=15682 ;=2.4(p=0.006)$ and the number of cigarettes $U=$ $16197 ;=.42(p=.007)$ is higher in men than in women.

Descriptors: Alcohol Drinking; Tobacco Use; Adolescent.

\section{Introducción}

El consumo de drogas es un problema de salud pública a nivel mundial que ha aumentado en los últimos veinte años, y es una de las causas principales que perturban la vida en comunidades y alteran la convivencia en las familias y la sociedad $^{(1)}$. El consumo de alcohol y tabaco como drogas lícitas representan un grave problema para los sistemas de salud en el mundo(2). La Organización Mundial de la Salud [OMS] en el 2014 señala que aproximadamente 3.3 millones de personas fallecieron por causas relacionadas con el alcohol en el mundo incluyendo a 320,000 jóvenes de 15 a 29 años de edad. De acuerdo con estas cifras el uso nocivo del alcohol genera el $5.9 \%$ de la mortalidad mundial, así como del $4.5 \%$ de la carga mundial de morbilidad expresada como pérdida de años de vida ajustados en función de discapacidad (3-4).

Según la Encuesta Nacional de Adicciones $(2011)^{5}$, cerca de $27,000,000$ de mexicanos consumen alcohol en edades entre 12 y 65 años y aunque el consumo no sea diario, se ha reportado que cerca de 4,000,000 de personas en México beben de forma excesiva una vez a la semana o con mayor frecuencia. El grupo de edad que presenta los niveles más altos de consumo es de 18 a 29 años de edad tanto en hombres como mujeres. Este hábito no es exclusivo de la población adulta; cada vez son más los adolescentes que se encuentran involucrados con el consumo y el abuso del alcohol, tanto que ha pasado a constituir una gran preocupación en los sectores de salud(6). 
Mientras tanto, el consumo de tabaco, es una de las principales causas prevenibles de enfermedad y muerte y es factor de riesgo de múltiples enfermedades coronarias y pulmonares tales como enfisema pulmonar, cáncer de pulmón, hipertensión arterial, angina de pecho, entre otros. En la actualidad $17,300,000$ mexicanos en edades de entre 12 y 65 años son fumadores activos, esto representa el $21.7 \%$ de la población, $12,000,000$ de hombres (31.4 $\%)$ y $5,200,000$ mujeres (12.6\%). El $8.9 \%$ de esta población fuma diariamente $(7,100,000)$ el $13.2 \%$ de hombres y el $4.8 \%$ de mujeres ${ }^{(5)}$.

En México cerca de 122 personas mueren a diario por causas que se vinculan con el tabaquismo; es causa probable de más de 25 enfermedades, y se estima que los fumadores crónicos pueden perder entre 20 y 25 años de vida. Este consumo ha generado discapacidades a corto, mediano y largo plazo, lo cual repercute en la economía y en el ámbito laboral, reflejando altos costos para el sector de salud por las enfermedades secundarias al consumo de tabaco y la disminución en la productividad de la población afectada causando la muerte en edad productiva ${ }^{(7)}$. Los estudios muestran que en México más de $60 \%$ de los fumadores inician su consumo antes de los 16 años de edad, y más de $90 \%$ lo hizo antes de los 20 años, lo que lleva a suponer que la ventana preventiva más importante se ubica en edades tempranas, ya que rara vez se informa el inicio de esta adicción en edades adultas ${ }^{(5,7)}$.

Aunado a esto los adolescentes se encuentran en una etapa de crecimiento y desarrollo, que los convierte en personas vulnerables al consumo del alcohol y tabaco, debido a que presentan tendencias a desafiar lo establecido y retar las normas sociales. Es por esto que existe la necesidad de continuar estudiando los patrones de consumo en los adolescentes, la edad de inicio de consumo y las diferencias entre sexo. El profesional de enfermería tiene un papel muy importante en la realización de medidas preventivas oportunas que puedan retrasar, evitar o reducir este consumo.

Por lo tanto el propósito del presente estudio fue identificar el consumo de alcohol y tabaco en los adolescentes.

\section{Materiales y Métodos}

El diseño del presente estudio fue descriptivo. La población estuvo conformada por 386 sujetos de entre 15 a 20 años de edad, de ambos sexos pertenecientes a una preparatoria de una Universidad Pública de un área suburbana del estado de Nuevo León. Se realizó un censo en un momento en el tiempo. Por lo que la muestra consistió en el total de los alumnos inscritos en la escuela preparatoria entre el primero y el tercer año $(n=386)$.

Se utilizó una Cédula de Datos Personales y Prevalencia de Consumo de Drogas Lícitas y el Cuestionario de Identificación de los Trastornos por Uso de Alcohol [AUDIT] $]^{(8)}$, que evalúa el tipo de consumo de alcohol en los últimos 12 meses. Los resultados oscilan de cero a cuarenta puntos, la calificación de uno a tres puntos se considera como consumo sensato (sin riesgo), el resultado de cuatro a siete puntos se considera como consumo dependiente (de riesgo) y el reporte de ocho a más puntos se considera como consumo dañino (perjudicial).

Los datos fueron procesados electrónicamente con el programa estadístico Statistical Package for the Social Sciences (SPSS) versión 18.0 para Windows. Se utilizó estadística descriptiva mediante frecuencias, proporciones, medidas de tendencia central y de variabilidad. Se realizó la prueba de bondad de ajuste de Kolmogorov-Smirnov con corrección de Lilliefors y se determinó la consistencia interna del instrumento a través del Coeficiente de Confiabilidad Alpha de Cronbach $(a=0.83)$

El presente estudio se apegó a lo dispuesto por el Reglamento de la Ley General de Salud en materia de Investigación para la Salud $(1987)^{(9)}$ en el cual se establece que para el desarrollo de investigación en salud, se deben contemplar aspectos éticos que garanticen la dignidad y el bienestar de los individuos. Para la realización de este estudio se contó con la aprobación de las Comisiones de Ética e Investigación de la Facultad de Enfermería de la Universidad Autónoma de Nuevo León, y se obtuvo por escrito la autorización de los directivos de la institución donde se llevó a cabo esta investigación. Se contactó a los estudiantes, una vez que aceptaron participar, se les entregó el consentimiento informado. Se entregó a cada participante un sobre cerrado con los instrumentos, se les corroboró el anonimato y la confidencialidad de la información.

\section{Resultados}

Las características sociodemográficas mostraron que, en cuanto al género predominó el sexo femenino en un $53.9 \%$, el $100 \%$ de los estudiantes eran solteros, el $48.7 \%$ cursaron el primer año de preparatoria y respecto a la ocupación el $91.2 \%$ solo estudiaba. 


\section{Oliva-Rodrìguez NN}

Los estudiantes presentaron una media de edad de inicio de consumo de alcohol de 14.1 años y una mediana de $14(D E=1.2)$, la edad de inicio de consumo de tabaco fue de 14.2 años y una mediana de 14 años $(D E=1.3)$. De acuerdo a la cantidad de bebidas consumidas en un día típico los participantes en promedio consumen 6 bebidas estándar $(D E=6.6)$ y consumen en promedio 2 cigarrillos por ocasión de consumo $(D E=2.4)$.

En la Tabla 1 se muestran las prevalencias del consumo de alcohol y tabaco, donde se puede observar que el $60.6 \%$ de los participantes han consumido alcohol y el $33.4 \%$ han consumido cigarrillos al menos una vez en la vida.

Tabla 1 - Prevalencia global, lápsica, actual e instantánea del consumo de alcohol y tabaco

\begin{tabular}{|c|c|c|c|c|c|c|c|c|}
\hline \multirow{3}{*}{ Prevalencia de Consumo } & \multicolumn{4}{|c|}{ Alcohol } & \multicolumn{4}{|c|}{ Tabaco } \\
\hline & \multicolumn{2}{|c|}{ Sí } & \multicolumn{2}{|c|}{ No } & \multicolumn{2}{|c|}{ Sí } & \multicolumn{2}{|c|}{ No } \\
\hline & $f$ & $\%$ & $f$ & $\%$ & $f$ & $\%$ & $f$ & $\%$ \\
\hline Alguna vez en la vida & 234 & 60.6 & 152 & 39.4 & 129 & 33.4 & 257 & 66.6 \\
\hline En el último año & 196 & 50.8 & 190 & 49.2 & 104 & 26.9 & 282 & 73.1 \\
\hline En el último mes & 138 & 35.8 & 248 & 64.2 & 74 & 19.2 & 312 & 80.8 \\
\hline En los últimos 7 días & 73 & 18.9 & 313 & 81.1 & 55 & 14.2 & 331 & 85.8 \\
\hline
\end{tabular}

$n=386$

En la Tabla 2 se presentan los tipos de consumo de alcohol reportados en el Cuestionario de Identificación de los Trastornos por Uso del Alcohol AUDIT, donde se observa que $21 \%$ de los participantes consumidores de alcohol muestra un consumo dañino.

Tabla 2 - Frecuencias y proporciones por tipo de consumo de alcohol según AUDIT

\begin{tabular}{lcc}
\hline \multicolumn{1}{c}{ Tipo de Consumo } & f & \% \\
\hline Consumo sensato & 75 & 19.4 \\
Consumo dependiente & 40 & 10.4 \\
Consumo dañino & 81 & 21.0 \\
\hline
\end{tabular}

Nota: $n=196$

Tabla 3 - Prueba U de Mann-Whitney para el índice de consumo de AUDIT por sexo

\begin{tabular}{|c|c|c|c|c|c|}
\hline & $n^{(\mathrm{a})}$ & $X^{(\mathrm{b})}$ & $M d n^{(\mathrm{c})}$ & $U^{(d)}$ & $p^{(\mathrm{e})}$ \\
\hline \multicolumn{6}{|c|}{ Índice de AUDIT } \\
\hline Femenino & 95 & 15.50 & 10.00 & 3771.5 & .009 \\
\hline Masculino & 101 & 21.56 & 20.00 & & \\
\hline \multicolumn{6}{|c|}{ Índice de Consumo Sensato } \\
\hline Femenino & 95 & 29.12 & 16.61 & 3642.5 & .003 \\
\hline Masculino & 101 & 39.36 & 33.33 & & \\
\hline \multicolumn{6}{|c|}{ Índice de consumo Dependiente } \\
\hline Femenino & 95 & 7.02 & 0.00 & 3819.0 & .006 \\
\hline Masculino & 101 & 13.78 & 8.33 & & \\
\hline \multicolumn{6}{|c|}{ Índice de Consumo Dañino } \\
\hline Femenino & 95 & 11.63 & 0.00 & 4480.0 & .393 \\
\hline Masculino & 101 & 14.05 & 6.25 & & \\
\hline
\end{tabular}

La Tabla 3 muestra diferencias significativas en el índice de AUDIT por sexo, siendo mayor en el sexo masculino que en el femenino $(p=.009)$. Se observan diferencias significativas entre mujeres y hombres en el índice de frecuencia y cantidad $(p=.003)$ y en el índice de consumo dependiente $(p=.006)$ siendo mayor en el sexo masculino, que en el femenino. Respecto a la cantidad de bebidas alcohólicas consumidas en un día típico, los hombres consumieron más bebidas que las mujeres mostrando una diferencia significativa $(p=$ .006). 


\begin{tabular}{|c|c|c|c|c|c|}
\hline & $n^{(\mathrm{a})}$ & $\bar{X}^{(b)}$ & $M d n^{(c)}$ & $U^{(d)}$ & $p^{(e)}$ \\
\hline \multicolumn{6}{|c|}{ Número de bebidas alcohólicas consumidas en un día típico } \\
\hline \multicolumn{6}{|l|}{ Femenino } \\
\hline \multirow[t]{2}{*}{ Masculino } & 208 & 2.24 & 0.00 & 15682.0 & .006 \\
\hline & 178 & 4.33 & 1.00 & & \\
\hline
\end{tabular}

(a) $n=196$

(b) $\mathrm{X}=$ media

(c)Mdn = mediana

(d) $U=U$ de Mann-Whitney

(e) $p=$ significancia

En la Tabla 4 se muestran los tipos de consumo de tabaco, donde se presenta que el $66.6 \%$ de los participantes nunca han fumado, el $14.2 \%$ Experimentadores, el $11.1 \%$ de los participantes son usuarios el $4.7 \%$ Ex Fumadores y el $3.4 \%$ Dependientes al tabaco.
Tabla 4 - Frecuencias y proporciones de los tipos de consumo de tabaco

\begin{tabular}{lrr}
\hline \multicolumn{1}{c}{ Tipo de Consumo de Tabaco } & \multicolumn{1}{c}{$\boldsymbol{f}$} & \multicolumn{1}{c}{$\%$} \\
\hline No Fumadores & 257 & 66.6 \\
Ex Fumadores & 18 & 4.7 \\
Experimentadores & 55 & 14.2 \\
Usuarios & 43 & 11.1 \\
Dependientes & 13 & 3.4 \\
\hline
\end{tabular}

$n=386$

La Tabla 5 muestra diferencia significativa en el número de cigarrillos consumidos en día típico por sexo, siendo mayor en el sexo masculino que en el femenino $(p=.007)$.

Tabla 5 - Prueba U de MannWhitney para el número de cigarrillos consumidos en un día típico por sexo

\begin{tabular}{lrrrrr}
\hline Número de cigarros consumidos en un día típico & $\boldsymbol{n}^{(a)}$ & $\overline{\boldsymbol{X}}^{(\text {b) }}$ & $\boldsymbol{M d}^{(c)}$ & $\boldsymbol{U}^{(\boldsymbol{d})}$ & $\boldsymbol{p}^{(e)}$ \\
\hline Sexo & & & & & \\
$\quad$ Femenino & 208 & .42 & .00 & 16197.0 & .007 \\
$\quad$ Masculino & 178 & 1.01 & .00 & & \\
\hline
\end{tabular}

(a) $n=386$,

(b) $\mathrm{X}=$ media

(c)Mdm= mediana

(d) $U=U$ de Mann-Whitney

(e) $p=$ significancia

\section{Discusión}

Se identificó que la edad de inicio de consumo de alcohol y tabaco fue a los 14 años de edad, el promedio de bebidas alcohólicas consumidas en un día típico fue de $6.3(D E=6.6)$, esto indica un consumo dependiente para hombres y consumo dañino para las mujeres ${ }^{(8)}$, probablemente este alto consumo de alcohol puede explicarse por el área donde residen, dado que están limitados a otras formas de distracción donde se puedan realizar actividades recreativas. Estos datos son sumamente alarmantes ya que en este grupo de edad cualquier cantidad de consumo se considera excesivo, debido a que puede ocasionar severos daños a corto plazo y desarrollar con mayor rapidez tolerancia y dependencia a esta sustancia por la etapa de desarrollo en la que se encuentran(10). Villatoro et al. (2011)(11) señalan que existe evidencia de que las drogas de inicio suelen ser el alcohol y el tabaco y a partir de este consumo pueden cambiar al consumo de sustancias ilícitas. Además entre más temprano inicia el consumo de sustancias, mayor es la probabilidad de aumentar la cantidad de consumo y de realizar la transición hacia el consumo de drogas ilícitas. 


\section{Oliva-Rodrìguez NN}

Respecto al número de cigarrillos consumidos en un día típico, el promedio de consumo fue de $2.5(D E=2.4)$ esto los ubica en la categoría de experimentadores, este hallazgo probablemente se explica por la influencia de los amigos, el deseo de experimentar riesgos, y las características propias de su edad que los llevan a retar y desafiar las normas sociales establecidas ${ }^{(12)}$.

Respecto la prevalencia global, lápsica, actual e instantánea del consumo de alcohol y tabaco en adolescentes de preparatoria muestra que la sustancia de mayor consumo es el alcohol y posteriormente el tabaco. Se presentaron proporciones de consumo alguna vez en la vida de $60.6 \%$ y $33.4 \%$ respectivamente.

Referente al consumo de alcohol y tabaco por sexo, se observan diferencias significativas con índice de AUDIT, las medias fueron más altas en los hombres, que en las mujeres, esto puede explicarse en las premisas socioculturales, donde es más aceptado el consumo de alcohol en hombres que en mujeres ${ }^{(13)}$, así mismo por la probable asociación de la sustancia como vehículo de socialización, y porque en adolescentes de sexo masculino se asocia este consumo con retar lo establecido, en cambio en las mujeres el consumo de alcohol puede ser socialmente no aceptado o estigmatizado ${ }^{(14)}$.

Para el consumo de tabaco, la cantidad de cigarrillos muestra diferencia significativa por sexo, las medias más altas se presentaron en los hombres, probablemente este hallazgo se explique por las creencias arraigadas que guardan las áreas suburbanas, donde el consumo de tabaco en las mujeres no es aceptado, por el contario es criticado socialmente.

Los datos del presente estudio apoyan los hechos de que el inicio del consumo de alcohol y tabaco es cada vez a edades más tempranas, además se soporta el hecho que los hombres adolescentes consumen más alcohol que las mujeres adolescentes y mayor cantidad y con mayor frecuencia.

\section{Conclusiones}

Predominó el sexo femenino en un $53.9 \%$. La media de edad de inicio de consumo de alcohol fue de 14.1 años, mientras que para el inicio de consumo de tabaco fue de 14.2 años. La cantidad de bebidas consumidas en un día típico fue de seis bebidas por ocasión y dos cigarros por ocasión. El $60 \%$ de los adolescentes habían ingerido alcohol al menos una vez en la vida y el $33.4 \%$ había consumido tabaco al menos una vez en la vida. El $21 \%$ de los bebedores presentaron un consumo dañino, el índice de AUDIT presentó diferencia significativa y fue mayor en los hombres que en las mujeres, el índice de frecuencia y cantidad, el índice de dependencia y la cantidad de bebidas ingeridas en un día típico también presentaron diferencias significativas, siendo mayor en hombres que en mujeres. El $66 \%$ de los adolescentes habían fumado al menos una vez en la vida, el $3.4 \%$ de los fumadores presentaron dependencia y los hombres consumen más cigarrillos por ocasión de consumo que las mujeres.

\section{Referencias}

1. Rodríguez PLA, Alonso CBA, Alonso CMM, Alonso CMT, Oliva RNN, Armendáriz GNA. Valores terminales, valores instrumentales y consumo de alcohol y tabaco en estudiantes de preparatoria. Enferm Comun. 2014;11(1):1-7.

2. Villegas, PMA, Alonso CMM, Alonso CBA, Martínez MR. Percepción de crianza parental y su relación con el inicio del consumo de drogas en adolescentes mexicanos. Aquichán. 2014;14(1):41-52.

3. Organización Mundial de la Salud. 2014 Global status report on alcohol and health. Géneve, 2014. ISBN 9789240692763.

4. Lema SL, Varela AM, Duarte AC, Bonilla GM. Influencia familiar y social en el consumo de alcohol en jóvenes universitarios. Rev Fac Nacional Salud Pública. 2011;29(3):264-71.

5. Consejo Nacional Contra las Adicciones-Secretaría de Salud [CONADIC-SS] Encuesta Nacional de Adicciones 2011. Cuernavaca, Morelos: Instituto Nacional de Salud Pública; 2011.

6. Armendáriz GNA, Alonso CMA, Alonso CBA, López CMA, Rodríguez PLA, Méndez RMD. La familia y el consumo de alcohol en estudiantes universitarios. Cienc Enferm. 2014;20(3):109-18.

7. Tapia CR. Las adicciones, dimensión, impacto y perspectivas. México, D.F: Manual Moderno; 2001.

8. De la Fuente JR, Kershenobich D. El alcoholismo como problema médico. Rev Fac Med UNAM. 1992;35(2):4751.

9. Secretaría de Salud. Reglamento de la Ley General de Salud en materia de Investigación para la Salud. 1987 [Acceso 13 Enero 2012]. Disponible en: http://www.salud. gob.mx/unidades/cdi/nom/compi/rlgsmis.html.

10. Secretaría de Salud. Norma Oficial Mexicana para la Prevención, Tratamiento y Control de las Adicciones, NOM-028-SSA2-1999. 1999. [Acceso 16 Octubre 2011]. Disponible en: http://www.unet.com.mx/ceca/norma.htm. 
11. Villatoro JA, Gaytán F, Moreno M, Gutiérrez ML, Oliva N, Bretón M. Tendencias del uso de drogas en la Ciudad de México: Encuesta de Estudiantes del 2009. Salud Mental. 2011;34(2):81-94.

12. Morardillo F. Los Valores, las Drogas, los adolescentes. Las Drogas Info. Instituto para el Estudio de las Adicciones. Ministerio de Sanidad, política social e igualdad. Gobierno de España. 2000. [Acceso 11 Marzo 2011]. Disponible en: http://www.lasdrogas.info/index.ph $\mathrm{p}$ ?op=InfoOpinion\&idOpinion=26.

13. Alonso CMM, Del Bosque MJ, Gómez MMV, Rodríguez AL, Esparza ASE, Alonso CBA. Percepción de normas sociales y consumo de alcohol en jóvenes universitarios. Anuario de Investigación en Adicciones. 2009;10(1):30-40.

14 Armendáriz GNA, Rodríguez AL, Guzmán FFR. Efecto de la autoestima sobre el consumo de tabaco y alcohol en adolescentes del área rural de Nuevo León, México. SMAD, Rev. Eletrônica Saúde Mental Álcool Drog. (Ed. port.) [Internet]. 2008 [Acceso 13 Enero 2016];4(1):1-15. Disponible en: http://pepsic. bvsalud.org/scielo.php?script=sci_arttext\&pid=S1806$69762008000100006 \&$ Ing=pt\&nrm=iso\&tlng=es 